Check for updates

Cite this: RSC Adv., 2017, 7, 27139

Received 15th March 2017

Accepted 10th May 2017

DOI: $10.1039 / \mathrm{c} 7 \mathrm{ra03079g}$

rsc.li/rsc-advances

\section{Binding of Euplotes octocarinatus centrin to peptide from xeroderma pigmentosum group $C$ protein (XPC) $\uparrow$}

\author{
Enxian Shi, ${ }^{\text {ab }}$ Wenlong Zhang, ${ }^{a}$ Yaqin Zhao (iD ${ }^{a}$ and Binsheng Yang (D) *a \\ Centrins are $\mathrm{Ca}^{2+}$-binding proteins that belong to the EF-hand (or calmodulin) superfamily, which are highly
} conserved among eukaryotes. To probe whether Euplotes octocarinatus centrin (EoCen) could replace human centrin 2 (HsCen2), herein, we chose a 22-residue peptide (K842-R863) from the human xeroderma pigmentosum group $\mathrm{C}$ protein (XPC), a dominant component of the nuclear excision repair (NER) pathway, and investigated the detailed structural and energetic characterization of the interaction with EoCen using spectrophotometric methods, native PAGE and isothermal titration calorimetry (ITC). Fluorescence and UV difference spectroscopy revealed that the well-conserved tryptophan residue was buried in the hydrophobic pocket exposed by the C-terminal domain of EoCen. The native PAGE indicated that a new band appeared corresponding to a complex which was exclusively mediated by $\mathrm{C}$ terminal domain of EoCen. Circular dichroism (CD) showed that peptide underwent a random coil-tohelix structural transition upon binding to the centrin, and ITC suggested centrin-peptide interactions were driven by an enthalpic contribution, which compensated for the unfavorable (negative) entropy term. Also, the affinity reduced by a factor of 4.67 compared with HsCen2, mainly due to V108 of the EoCen substitution for $\mathrm{L} 112$ of HsCen2.

\section{Introduction}

Centrins are small $(\sim 20 \mathrm{kDa})$, acidic, $\mathrm{Ca}^{2+}$-binding proteins that belong to the EF-hand calmodulin (CaM) superfamily. The first members of centrins to be discovered were in the unicellular green algae Tetraselmis striata ${ }^{1}$ and Chlamydomonas reinhardtii, as major components of the $\mathrm{Ca}^{2+}$-sensitive contracting fibers associated with the basal bodies. ${ }^{2,3}$ Afterwards orthologous centrins were also observed in many eukaryotes, e.g. yeast, ${ }^{4}$ higher plants, ${ }^{5}$ invertebrates ${ }^{6}$ and humans. ${ }^{7,8}$ Only one isoform existed in the lower eukaryotes, such as green algae and yeast; however, four isoforms have been discovered in the higher eukaryotes. Only about $10 \%$ (ref. 9) of the centrins are concentrated on the microtubule organizer centers (MTOC), which are structurally and functionally similar to basal bodies in ciliated and flagellated cells, the spindle pole bodies (SPB) in yeast and the centrosome in higher eukaryotes. Centrins regulate the duplication and segregation of MTOC during the cell cycle. ${ }^{10,11}$ In addition, the majority of centrins are localized in the nucleus region, where they play diverse roles. HsCen2 has been

${ }^{a}$ Institute of Molecular Science, Key Laboratory of Chemical Biology of Molecular Engineering of Education Ministry, Shanxi University, Taiyuan 030006, PR China. E-mail: yangbs@sxu.edu.cn; Fax: +86-351-7016358

${ }^{b}$ Department of Pharmacy, Shanxi Medical University, Taiyuan 030001, PR China

$\uparrow$ Electronic supplementary information (ESI) available. See DOI: 10.1039/c7ra03079g demonstrated to participate in the nuclear excision repair (NER) inside the nucleus, ${ }^{12,13}$ and in the export of mRNA from the nucleus. ${ }^{14}$ Other possible implications were also found in the regulation of the G-protein transducin (Gt) in photoreceptor cells ${ }^{15}$ or in the activity of the paramecium ciliary reversalcoupled voltage-gated $\mathrm{Ca}^{2+}$ channels. ${ }^{16}$

The biological functions of centrins require an association with diverse protein targets in various cellular processes. In yeast $\mathrm{SPB}$, centrins interact with three proteins in the half-bridge,

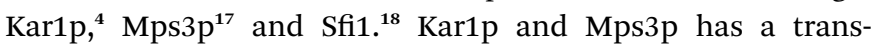
membrane domain and are likely involved with the lipid bilayer of the half-bridge. However, Sfi1, which plays a critical role in SPB duplication and has $\sim 20$ centrin-binding motifs, does not have a transmembrane domain. XPC, ${ }^{12}$ which possesses only one centrin-binding site, triggers NER and recognizes bulk DNA lesions. The yeast complex of Sac3 (ref. 19) with Sus1 and Cdc31 promotes nuclear pore association and mRNA export. Transducin, a G-protein from visual signal transduction, is also a potential centrin target in mammalian photoreceptor cells. ${ }^{15}$ Furthermore, HsCen2, HsCen3 and EoCen can bind to melittin, a natural peptide from bee venom mimicking CaM targets. ${ }^{20-23}$ Centrins bind targets mainly through the hydrophobic triad $\mathrm{W}^{1} \mathrm{xxL}^{4} \mathrm{xxxL}^{8}$, which was first identified in XPC. However, the binding motif is in the opposite orientation, $\mathrm{L}^{8} \mathrm{xxxL}^{4} \mathrm{xxW}^{1}$, in centrin's other targets (Sac3, Sfi1 and transducin).

Ciliate Euplotes octocarinatus centrin (EoCen) was first reported by our laboratory ${ }^{24}$ and is cloned from E. octocarinatus; 
the precise biological functions remain to be determined. EoCen is a protein of 168 residues, which shares about 60, 62 and $66 \%$ sequence identity with HsCen1, HsCen 2 and HsCen3, respectively, and shares approximately 50\% sequence identity with the well-studied EF-hand protein CaM. Like CaM, centrin is organized into two independent domains tethered by a central linker, each domain consisting of a pair of EF-hand motifs of helix-loop-helix that can potentially bind two $\mathrm{Ca}^{2+}$ ions. ${ }^{25}$ Sequence analysis and the available experimental data suggest that the diversity among various centrins stems from the first 20-residue fragment and this stretch is absent in standard CaM. $^{26}$ Earlier biochemical and physicochemical experiments have demonstrated that the N-terminal fragment plays a critical role in self-assembly, ${ }^{27-30}$ whereas the C-terminal fragment is strongly conserved and contributes to the target recognition of centrin. ${ }^{31-33}$ Our group have reported that the melittin binding is mainly mediated by the C-terminal fragment of EoCen $\left(K_{\mathrm{a}} \sim 10^{6} \mathrm{M}^{-1}\right) .{ }^{22,23,34}$

Human heterodimer XPC/hHR23B behaved as a damage detector that initiated the global genome NER, ${ }^{35}$ and centrin 2 interacts with the heterodimer physically and stimulates the NER process. ${ }^{13}$ In Arabidopsis thaliana, the homolog of Atcen2 and AtRad4 interacted to modulate the repair efficiency of bulk DNA lesions. ${ }^{36}$ Also, the yeast ortholog Rad4-Rad23 and human XPC-Rad23B showed almost equal binding affinities to the damaged DNA duplex, and recognized DNA lesions in analogous methods. ${ }^{37}$ The NER protein Rad33 showed a functional resemblance to the centrin homologue Cdc31. ${ }^{38}$ However, the XPC homologue in Euplotes octocarinatus has not been identified yet. The centrin-binding motif of XPC homologs from species were strongly conserved (see Fig. 6B), so we chose a 22residue synthetic peptide derived from human XPC to study the peptide recognition of EoCen. Whether P22-XPC could substitute Euplotes octocarinatus XPC protein or EoCen could substitute HsCen 2 may be the first step to understand the possibility of NER in Euplotes octocarinatus. We characterized the structural and energetic aspects of the interaction and explained the lower affinity compared with HsCen 2 from the amino acid sequence. Our results revealed biochemical features of centrin binding to XPC and provide insights into the molecular mechanism of centrin functions in the cellular context.

\section{Experimental}

\subsection{Reagents}

Ethylene diamine tetraacetic acid (EDTA), 4-(2-hydroxyethyl)-1piperazineethane sulfonic acid (Hepes), potassium chloride and other solvents were all analytic grade reagents. L-Tryptophan was chromatographically pure.

\subsection{Protein expression and purification}

For the expression and purification of intact Euplotes octocarinatus centrin, the $\mathrm{N}$-terminal domain and $\mathrm{C}$-terminal domains were carried out according to our previous publications. ${ }^{24}$ After purification, the proteins were kept at $-20{ }^{\circ} \mathrm{C}$, and the stock protein solutions were conserved in $10 \mathrm{mM}$ Hepes, $100 \mathrm{mM} \mathrm{KCl}$, pH 7.4.

\subsection{Peptide synthesis}

The peptide derived from human xeroderma pigmentosum group C (P22-XPC; KRALGNWKLLAKGLLIRERLKR), Lys842Arg863 (ref. 31 and 39) covering the crucial centrin-binding region was synthesized by Wuhan Fine Peptide Co., Ltd. The purity of the XPC peptide was greater than $98 \%$, as indicated by HPLC. The molecular mass was verified by microelectrospray ionization mass spectrometry.

\subsection{UV spectroscopy}

UV spectra were recorded using a Varian Cary 50 Bio UV-Visible Spectrophotometer. Each decalcified protein was diluted to $10 \mathrm{mM}$ Hepes, $100 \mathrm{mM} \mathrm{KCl}, \mathrm{pH}$ 7.4. The peptide concentration was assessed spectrophotometrically using an extinction coefficient of $5500 \mathrm{M}^{-1} \mathrm{~cm}^{-1}$ at $280 \mathrm{~nm}$. The protein concentration was determined using an extinction coefficients of 5600 (EoCen) $\mathrm{M}^{-1} \mathrm{~cm}^{-1}$ and of 1400 (C-EoCen) and 4350 (N-EoCen) $\mathrm{M}^{-1} \mathrm{~cm}^{-1}$ at $280 \mathrm{~nm}$. The sample included $77.4 \mu \mathrm{M}$ of individual compounds or of the equimolar mixtures. The concentration of tryptophan in different solvents was $55 \mu \mathrm{M}$.

\subsection{Fluorescence emission spectroscopy}

Binding of the XPC peptide to EoCen, N-EoCen, and C-EoCen was monitored by fluorescence spectroscopy. The experiments were performed on a Fluoromax-4 spectrofluorometer (HORIBA Jobin Yvon, France). The excitation wavelength was set at $280 \mathrm{~nm}$, and fluorescence emission spectra were recorded from 295 to $450 \mathrm{~nm}$, with all slit widths set to $5 \mathrm{~nm}$, using quartz cuvettes. The sample buffer contained $10 \mathrm{mM}$ Hepes, $100 \mathrm{mM}$ $\mathrm{KCl}, \mathrm{pH}$ 7.4. The sample included $8 \mu \mathrm{M}$ of individual compounds or of equimolar mixtures. To attenuate the interference from the tyrosine residue in EoCen, the excitation wavelength was set at $295 \mathrm{~nm}$ in the titration experiments. Aliquots of a $480 \mu \mathrm{M}$ protein sample were added into an XPC peptide solution $8 \mu \mathrm{M}$ (initial concentration) and incubated for $3 \mathrm{~min}$. The emission spectrum was recorded over the range 305$450 \mathrm{~nm}$. About 20 data points were collected per titration over protein-peptide molar ratios of $0-2$. To correct the dilution effect, the fluorescence intensity was converted to molar fluorescence intensity by dividing the fluorescence intensity by the analytical concentration of the XPC peptide. Also, changes in the fluorescence intensity at $331 \mathrm{~nm}$ were assigned to the complex and the data were fitted to a single-site binding model using SigmaPlot 10.0 (shown in ESI $\dagger$ ).

\subsection{Gel electrophoresis}

The electrophoretic mobility of the protein was evaluated by nondenaturing polyacrylamide gel electrophoresis (PAGE) under discontinuous conditions as a modified technique described by Beckingham. ${ }^{\mathbf{4 0}}$ Polyacrylamide gels contained $390 \mathrm{mM}$ Tris ( $\mathrm{pH}$ 8.8), 0.1\% ammonium persulfate, and 15\% acrylamide/bis (29:1). To induce polymerization, 0.1\% TEMED 
was added. A tris-glycine electrophoresis running buffer was composed of $25 \mathrm{mM}$ Tris $(\mathrm{pH} 8.3)$ and $250 \mathrm{mM}$ glycine. All electrophoreses were performed at room temperature. The gels were run at a constant current of 11-12 mA for $2 \mathrm{~h}$. The gels were stained with Coomassie blue R-250 for $5 \mathrm{~min}$, and washed in distilled water for $2 \mathrm{~h}$.

\subsection{Isothermal titration calorimetry (ITC)}

The thermodynamic parameters of the molecular interactions between EoCen (intact EoCen and two isolated domains) and target peptide (P22-XPC) at $30{ }^{\circ} \mathrm{C}$ were investigated by ITC using a MicroCal iTC200 instrument (Malvern Instruments Ltd. UK). The proteins and peptide in the presence of EDTA were equilibrated in the same buffer (10 mM Hepes, $100 \mathrm{mM} \mathrm{KCl,} \mathrm{pH} \mathrm{7.4).}$ The centrins $(77.1 \mu \mathrm{M})$ in the calorimeter cell were titrated with the target peptide $(1540 \mu \mathrm{M})$ by successive automatic injections of $0.5 \mu \mathrm{L}$ each. The first injection of $0.2 \mu \mathrm{L}$ was ignored in the final data analysis. Integration of the peaks corresponding to each injection and a correction for baseline were performed using the Origin-based software provided by the manufacturer. The data were fit to an interaction model to generate the stoichiometry $(n)$, equilibrium binding constant $\left(K_{\mathrm{a}}\right)$, and enthalpy of complex formation $(\Delta H)$. The free energy $(\Delta G)$ was determined using the following equations: $\Delta G=-R T \ln \left(K_{\mathrm{a}}\right)$, where $R(1.987$ cal $\left.\mathrm{mol}^{-1}\right)$ is the gas constant and $T(303.15 \mathrm{~K})$ is the absolute temperature, and $\Delta G=\Delta H-T \Delta S$. The following units were used: affinity constant $\left(K_{\mathrm{a}}\right) \mathrm{M}^{-1}$; Gibbs energy change $(\Delta G)$ kcal $\mathrm{mol}^{-1}$; enthalpy change $(\Delta H) \mathrm{kcal} \mathrm{mol}^{-1}$; and entropy change $(\Delta S) \mathrm{kcal}(\mathrm{mol} \mathrm{K})^{-1}$. Titrations were performed in triplicate. To determine the dilution/mixing heat, control experiments was performed by injecting the peptide solution into the buffer.

\subsection{Far-UV circular dichroism (CD)}

CD experiments were performed on a Chirascan spectrometer (Applied Photophysics Ltd. UK) continuously purged by $\mathrm{N}_{2}$ and equipped with a temperature-control system. Far-UV spectra were recorded between 200 and $250 \mathrm{~nm}$ using $1 \mathrm{~mm}$ quartz cells. The spectra were collected as an average of three scans with a step size of $1 \mathrm{~nm}$ and a bandwidth of $1 \mathrm{~nm}$. The samples were dissolved in $10 \mathrm{mM}$ Hepes, and $100 \mathrm{mM} \mathrm{KCl} \mathrm{(pH} \mathrm{7.4).} \mathrm{The}$ concentrations of the full-length EoCen, C-terminal domain and $\mathrm{N}$-terminal domain were all $7.84 \mu \mathrm{M}$. The buffer signal was digitally subtracted using software provided by the manufacturer. For the P22-XPC titration, small aliquots of $0.47 \mathrm{mM}$ peptide solution were added to achieve an XPC peptide to proteins molar ratio of $0.25-2$. To correct for dilution and to normalize the results from different titrations, the CD data were converted to molar ellipticity by dividing the analytical concentration of protein.

\section{Results and discussion}

\subsection{Interaction with XPC peptide monitored by micro- environment change of Trp}

In proteins, the Trp residue is often regarded as an intrinsic fluorescent probe to monitor protein conformational change, because the indole chromophore is very sensitive to changes in the surrounding microenvironment. ${ }^{41}$ The XPC peptide contains only one tryptophan residue, whereas EoCen includes no tryptophan and has four tyrosine residues which are not sensitive to the micro-environment. The maximum emission wavelength of the peptide alone was situated at $356 \mathrm{~nm}$ (line b in Fig. $1 \mathrm{~A}-\mathrm{C}$ ), reflecting the exposure of the indole moiety to the highly polar environment. Adding 1 equivalent of XPC peptide to the intact protein or its C-terminal fragment solution resulted in 1.65-fold and 1.40-fold increases in intensity, respectively, accompanied by a blue-shift (from 356 to $331 \mathrm{~nm}$ ) of the emission maximum, which manifested that the complex was formed in the absence of $\mathrm{Ca}^{2+}$ ion (that is, in the presence of EDTA). At the same time, fluorescence spectra of the intact protein as well as the C-terminal fragment were scanned as a control (line a in Fig. 1A and B), respectively. However, for the $\mathrm{N}$-terminal fragment, the maximum emission of the equimolar mixture remained unchanged, which suggested that the $\mathrm{N}$ terminal was not significantly engaged in peptide binding. The environmental sensitivity of the fluorescence parameters of the peptide binding to centrin strongly suggested that the indole fluorophore was oriented in a hydrophobic cavity exposed by the protein, resembling other Trp-containing target peptides bound to $\mathrm{CaM}^{42}$ or centrin..$^{20,43-45}$ Moreover, the fluorescence intensity and the shape of the emission spectra still kept unchanged even when the salt concentrations reached $2 \mathrm{M}$ (Fig. S1C $\dagger$ ), which implied that the complex formation was mainly mediated by a hydrophobic interaction and the electrostatic interaction played negligible role.

Successive titration proteins to XPC peptide solution resulted in an increase in intensity at $331 \mathrm{~nm}$ and an isosbestic point appeared at 378 and $367 \mathrm{~nm}$ for EoCen and C-EoCen, respectively (Fig. S1A and $\mathrm{B} \dagger$ ). The isosbestic point indicated that there were only two distinct species in the system, namely the XPC peptide and complex. The Trp fluorescence change represented the formation of a $1: 1$ complex (inset of Fig. S1A and $\mathrm{B} \dagger$ ). The binding isotherms were obtained by changes in intensity at $331 \mathrm{~nm} v s$. the free concentrations of protein (Fig. 1D) and were well fitted to a one-site model using an iteration method (Fig. S2 $\dagger$ ) and produced a dissociation constant $K_{\mathrm{d}}$ of $(0.22 \pm$ $0.01)$ and $(0.17 \pm 0.01) \mu \mathrm{M}$ for the C-terminal fragment and intact protein, respectively (see ESI $\dagger$ ), almost in agreement with the values obtained by calorimetry titrations (Table 2).

Additional support for this structural detail was provided by UV difference spectra of tryptophan residue. EoCen contains no cysteine or tryptophan and has four tyrosine residues and ten phenylalanine residues, so UV spectra of intact and isolated fragments of EoCen were characteristic of tyrosine and phenylalanine (line $\mathrm{b}$ in Fig. S3A-C $\dagger$ ). The absorption bands at 253, 259, 265 and $269 \mathrm{~nm}$ were due to the absorption by Phe, ${ }^{20}$ and those at 279 and $286 \mathrm{~nm}$ were attributed to the absorption of Tyr. ${ }^{46,47}$ The XPC peptide contains only one tryptophan, so the peptide showed the representative profile of tryptophan (the absorption wavelengths were at 280 and $290 \mathrm{~nm}$ ) (line c in Fig. S3A-C $\dagger$ ). It is important to emphasize that absorption at about $290 \mathrm{~nm}$ is only for the tryptophan residue and ruled out contributions from phenylalanines or tyrosines in protein. 

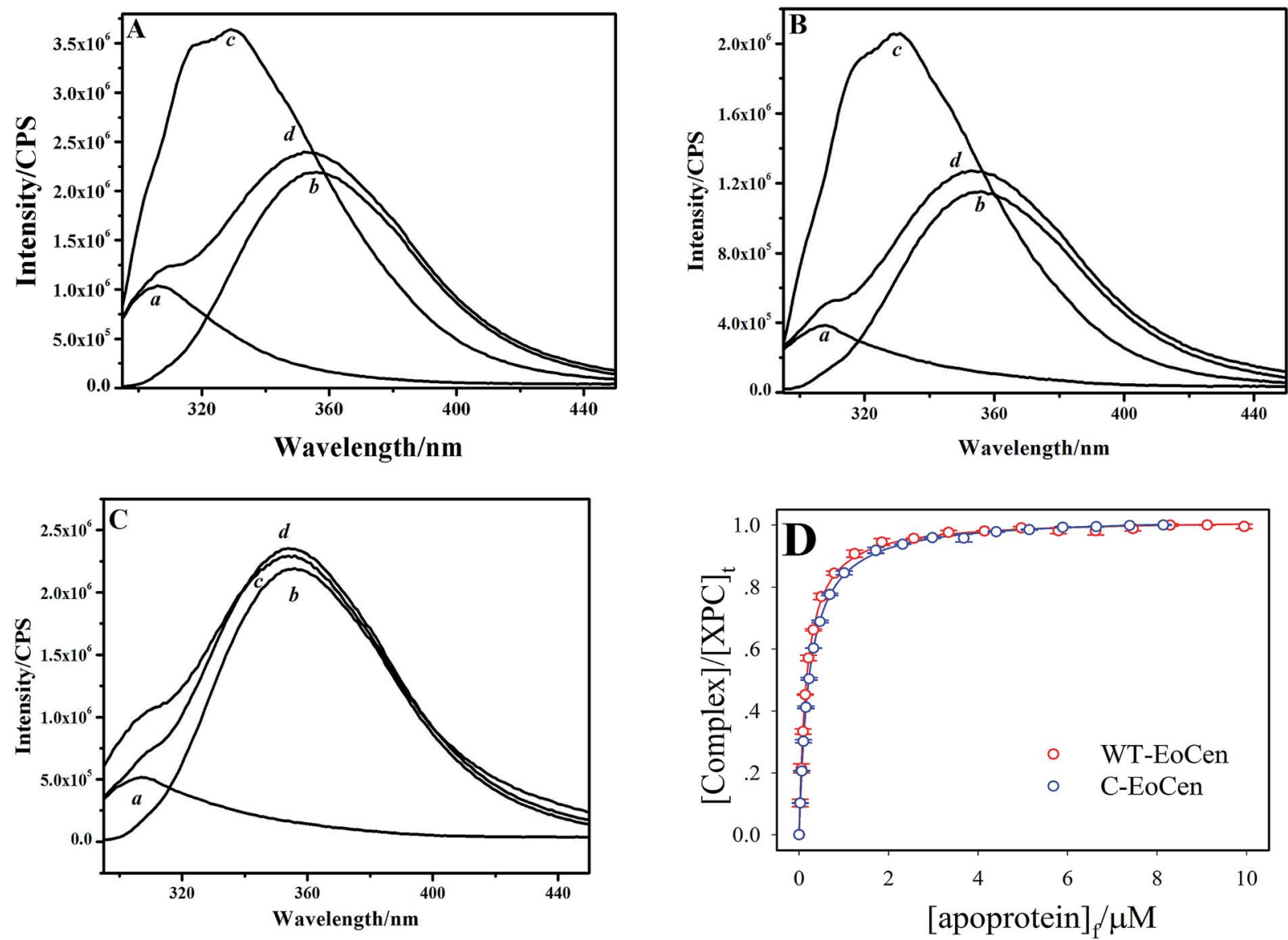

Fig. 1 Interaction between the XPC peptide and apoprotein measured by the Trp fluorescence of XPC in $10 \mathrm{mM} \mathrm{Hepes,} 100 \mathrm{mM} \mathrm{KCl}(\mathrm{pH} 7.4)$ in the presence of EDTA for WT-EoCen (A), C-EoCen (B), and N-EoCen (C). Samples contain $2 \mathrm{~mL}$ of $8 \mu \mathrm{M}$ of the individual compounds or of the equimolar mixture. (a) Protein, (b) XPC peptide, (c) complex of protein/XPC, (d) the calculated sum spectra of same concentrations of protein and XPC peptide. Binding isotherms for the interaction of XPC peptide with EoCen (red) and C-EoCen (blue) (D). Data points are an average of three experiments. Solid lines represent the best fits, according to eqn (4) in ESI. $\dagger$
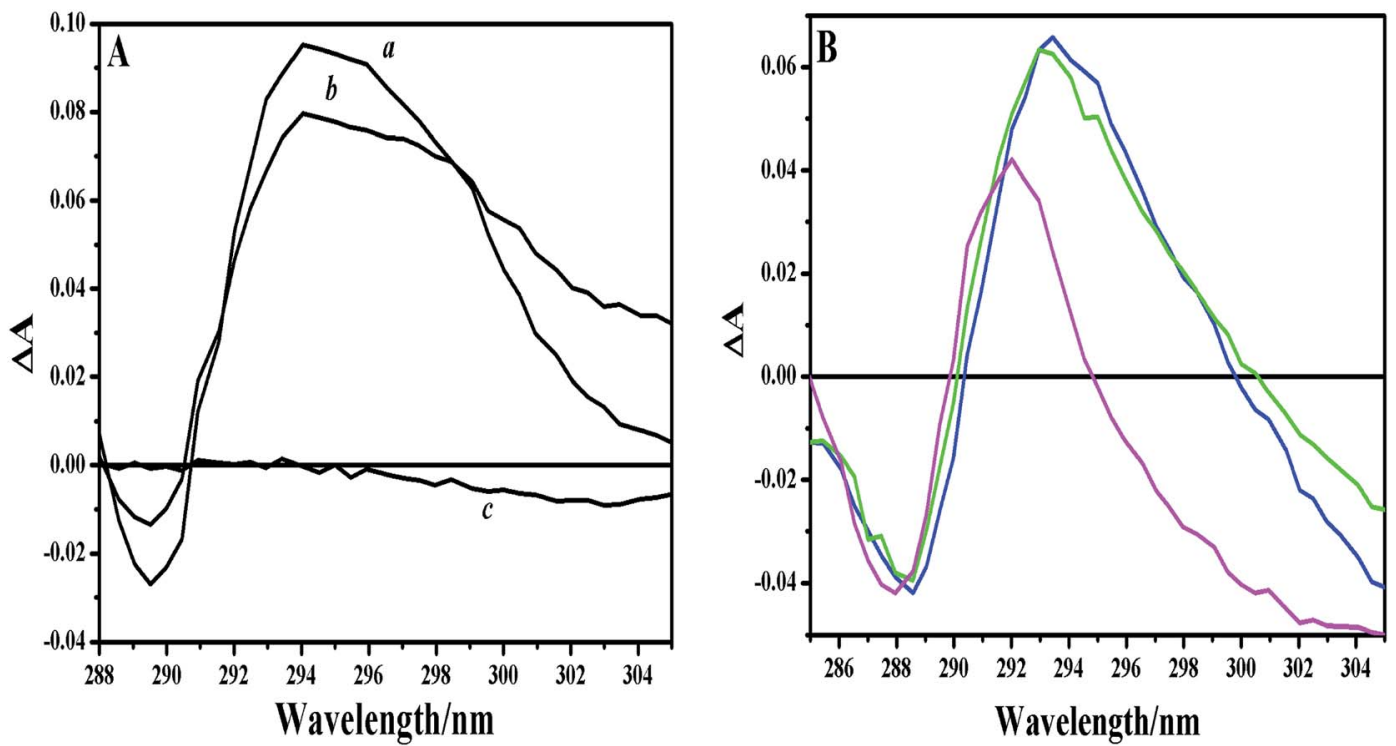

Fig. 2 (A) Difference UV spectra of XPC bound to proteins in $10 \mathrm{mM} \mathrm{Hepes,} 100 \mathrm{mM} \mathrm{KCl}$, pH 7.4 (using the calculated sum spectra of the proteins and the XPC peptide as reference). Integral EoCen (a), C-EoCen (b), N-EoCen (c). (B) Difference UV spectra of tryptophan in different solvents (using tryptophan in water as blank). DMSO (blue line), DMF (green line), dioxane (magenta line). 
However, there were no apparent differences between the spectra of equimolar mixtures and the sum of the spectra of individual components. To highlight the alteration to the
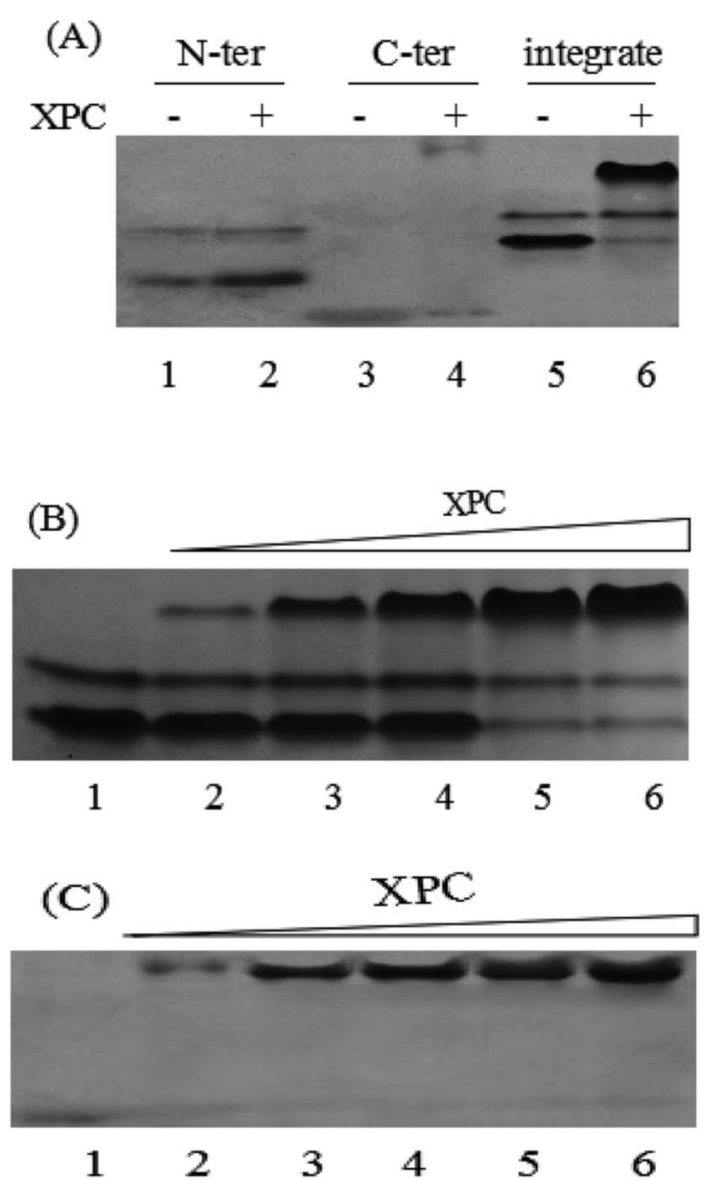

Fig. 3 (A) Native PAGE of XPC peptide binding to N-EoCen, C-EoCen and WT-EoCen in the presence of EDTA. The concentrations of NEoCen and C-EoCen were $100 \mu \mathrm{M}$, and that of WT-EoCen was $60 \mu \mathrm{M}$. The ratio of the XPC peptide to protein was $1: 1$. (B) Concentration gradient of XPC peptide binding to WT-EoCen by means of native PAGE in the presence of EDTA. The concentration of WT-EoCen is 120 $\mu \mathrm{M}$. The ratio of XPC to WT-EoCen is 0.0 (1), 0.2 (2), 0.5 (3), 0.8 (4), 1.0 (5), 2.0 (6). (C) Concentration gradient of XPC peptide binding to CEoCen by means of native PAGE in the presence of EDTA. The concentration of $\mathrm{C}$-EoCen is $500 \mu \mathrm{M}$. The ratio of XPC to $\mathrm{C}$-EoCen is 0.0 (1), 0.3 (2), $0.5(3), 0.8(4), 1.0(5), 1.3(6)$. environment of tryptophan in the peptide, UV difference spectra were obtained by subtracting the calculated sum from experimental values (Fig. 2A). A positive peak can be seen centered about $294 \mathrm{~nm}$ and a negative peak situated at $289 \mathrm{~nm}$ for the intact protein and the C-terminal domain, indicating the change of environment around Trp. To verify the effect, UV spectra of tryptophan in different solvents were performed (Fig. S3D $\dagger$ ). The absorption wavelength of tryptophan in $\mathrm{H}_{2} \mathrm{O}$ was located at $288 \mathrm{~nm}$, and shifted to $291 \mathrm{~nm}$ in each of DMSO, DMF, and dioxane. The UV difference spectra of tryptophan in different solvents showed an increase in absorbance as the positive peaks centered at about $293 \mathrm{~nm}$ and a decrease in absorbance as the negative peaks situated at $289 \mathrm{~nm}$ (Fig. 2B). The control experiment demonstrated that the shifts of peaks resulted from the increase in apolarity of the microenvironment around Trp. This was firstly demonstrated by our group although UV spectra were less sensitive than fluorescence spectra. ${ }^{\mathbf{4 1}}$ In contrast, there was no change when the $\mathrm{N}$ terminal fragment was incubated with XPC peptide, indicating no measurable interaction. So, a conclusion can also be drawn that the micro-environment around the Trp residue in the peptide changed from a polar water solvent to a more hydrophobic interface upon complex formation.

\subsection{Native PAGE assay}

In our research, the complex formation of EoCen with the peptide was monitored on the native PAGE, which is possibly the most highly resolving electrophoretic method yet established for separating proteins. The centrin superfamily is characterized by an additional N-terminal segment of about 20 residues with a basic property and high sequence diversity, which are absent in calmodulin and other $\mathrm{Ca}^{2+}$-binding proteins. ${ }^{26}$ It is the distinction between centrin and CaM that result in the centrins' unique character, namely aggregation, as has been corroborated by our group. ${ }^{\mathbf{2 9 , 3 0}}$ From Fig. 3A (lane 1 and lane 5), two bands with different mobilities for the full length and N-terminal domain of EoCen could be observed, which implied two distinct conformations of the protein. The bottom band that migrated faster contributed to the monomers, and the top clear band that migrated more slowly corresponded to the multimers. By contrast, the C-terminal domain had less tendency to self-association and possessed a smaller molecule weight and exhibited a much more negative net

Table 1 Parameters of apoprotein and its complex with the XPC peptide ${ }^{a}$

\begin{tabular}{|c|c|c|c|c|c|c|}
\hline XPC & 22 & 2.6 & 12.02 & 1 & 8 & +7 \\
\hline Cen-XPC $(1: 1)$ & 190 & 22.3 & 5.15 & 40 & 34 & -6 \\
\hline C-Cen & 79 & 9.2 & 4.47 & 20 & 12 & -8 \\
\hline C-Cen-XPC (1:1) & 101 & 11.8 & 5.60 & 21 & 20 & -1 \\
\hline
\end{tabular}

${ }^{a}$ Data available on the web site http://web.expasy.org/protparam/. 

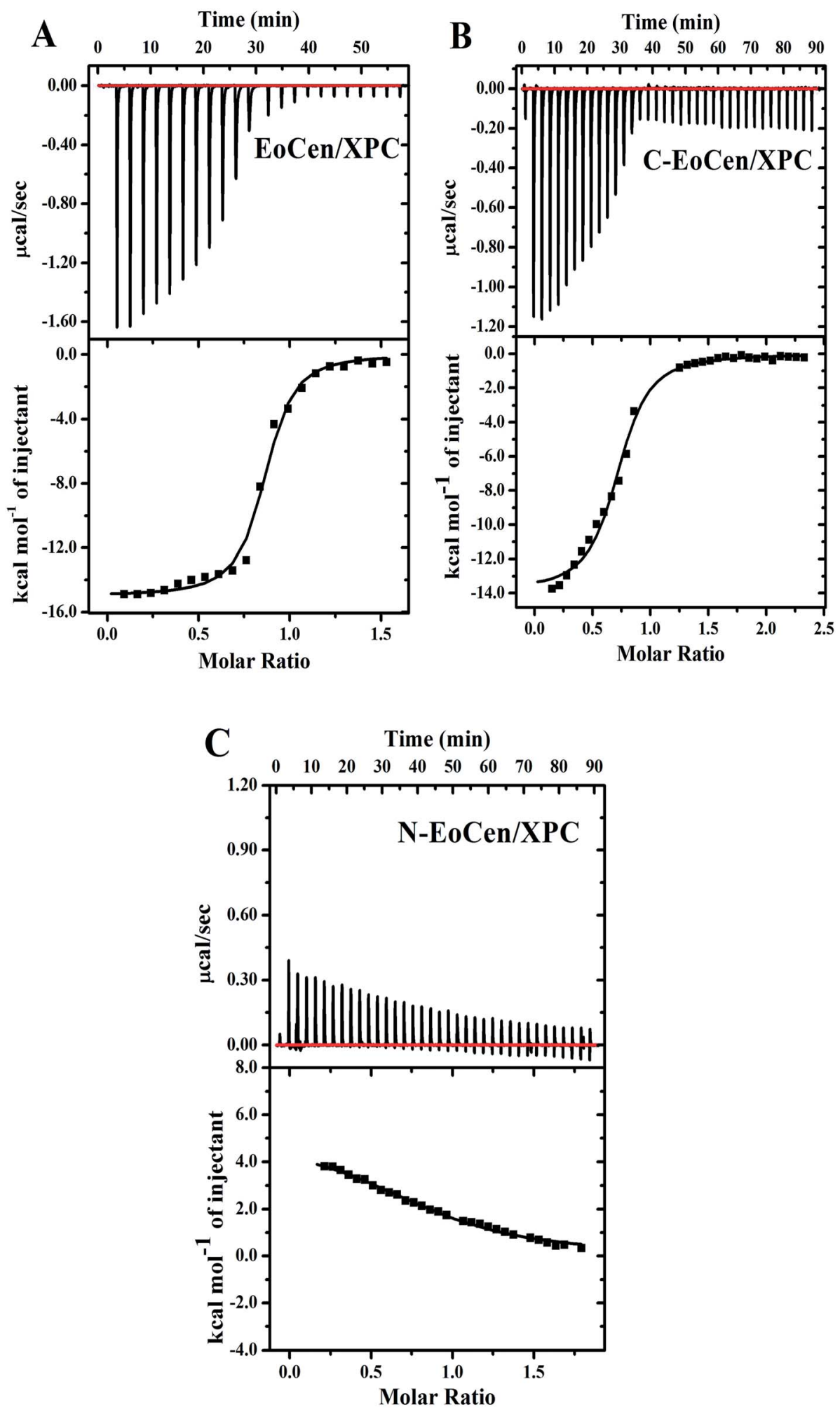

Fig. 4 Peptide binding to WT-EoCen (A), C-EoCen (B), and N-EoCen (C), studied by isothermal titration calorimetry. Thermograms (top panels) and binding isotherms (bottom panels) of the titration of the XPC peptide, in the absence of $\mathrm{Ca}^{2+}$, at $30{ }^{\circ} \mathrm{C}$.

charge among these proteins, so the position of it was much closer to the bottom of the gel (the positive pole) (Fig. 3A lane 3).

After apoprotein was incubated with the XPC peptide with a ratio of $1: 1$ at $4{ }^{\circ} \mathrm{C}$ overnight, the sample was analyzed by native PAGE. It could be seen that a clear new band appeared with a mobility slightly slower than that of EoCen, indicating the formation of the peptide-centrin complex, among which the positively charged XPC peptide alone could not migrate to the 
anode in this native system. And the band of monomers and multimers faded simultaneously (Fig. 3A lane 6), suggesting the addition of the peptide inhibited the centrin's aggregation. Similar observations occurred for the C-terminal domain of EoCen. The complex of C-EoCen and the XPC peptide with increasing molecule weight and decreasing negative net charge from 8 to 1 comparing with C-EoCen, was positioned at the top of the gel, migrating most slowly. In addition, the interaction between the XPC peptide and N-EoCen was also investigated. As shown in Fig. 3A (lane 2), no new complex band could be observed even though the ratio of XPC peptide to N-EoCen reached 5.0 and the reaction time extended to $48 \mathrm{~h}$ (data not shown). The parameters of EoCen and its complex are listed in Table 1. EoCen and C-EoCen were acidic proteins, having a lower pI value (between 4.40 and 4.90 ), and the XPC peptide contained 8 basic residues, having a higher $\mathrm{pI}$ value (12.02). As alluded to above, the interaction between EoCen and XPC was mediated exclusively by the C-terminal domain. Although the net charge of the $\mathrm{N}$-terminal domain is -6 , no complex formed with the XPC peptide, proving that the hydrophobic interaction dominated without a significant electrostatic component. With the increasing ratio of peptide to apoprotein, the complex band became highlighted and the monomers and multimers faded away gradually (Fig. 3B and C). The new band reached a maximal intensity at an equimolar mixture of both proteins. Native PAGE was a visual inspection to detect the existence of the complex.

\subsection{High-affinity binding of XPC peptide to EoCen}

The binding properties of the P22-XPC peptide to EoCen were investigated using ITC, an efficient method for the determination of the affinity constant and the energetic balance of molecular interactions. The raw ITC data and the binding isotherms corresponding to the interaction of the XPC peptide with apoEoCen and its two isolated domains are shown in Fig. 4, and the total thermodynamics parameters for the three proteins are presented in Table 2. For the integral protein, the change in enthalpy $(\Delta H)$ was negative, indicating that the binding reaction was exothermic. Under the experimental conditions (in the presence of EDTA), the isotherm for the integral protein indicated a single binding event with a stoichiometry of $1: 1$ and a high affinity $K_{\mathrm{a}}=(1.67 \pm 0.35) \times 10^{6} \mathrm{M}^{-1}$. The large reaction enthalpy change greatly overcame the entropy component to the free energy of interaction. The metalfree form of C-EoCen interacted with the peptide with a similar affinity $\left((0.46 \pm 0.09) \times 10^{6} \mathrm{M}^{-1}\right)$ and the binding was enthalpically driven $\left(\Delta H=-13.88 \mathrm{kcal} \mathrm{mol}^{-1}\right.$, at $\left.30^{\circ} \mathrm{C}\right)$. The association constant for the isolated C-EoCen was 3.6-fold smaller than that of the high-affinity site in the integral protein in the present conditions. The stoichiometry was also one, meaning that the C-terminal domain of EoCen had only one site for XPC. These results confirmed that the C-terminal domain of EoCen could bind the target even in the absence of $\mathrm{Ca}^{2+}$ ions. Indeed, the free energy of the peptide binding to C-EoCen contributed $92 \%$ of that of the integral protein.

In the case of N-EoCen, the heating rate was very small, and remained nearly constant during the titration, failing to show a detectable interaction in the present conditions. ITC measurements revealed again that the unique binding site in apoEoCen was situated in the C-terminal domain and the centrin-peptide interactions were driven by the enthalpy contribution, which compensated for the unfavorable (negative) entropy component.

\subsection{Conformational change of the interaction}

CD spectroscopy was used as an elementary investigation tool to characterize the conformation properties of the EoCen/peptide interaction. As shown in Fig. 5, the far-UV CD spectrum of apoproteins showed two negative bands at 222 and $208 \mathrm{~nm},{ }^{48}$ which is typical for a well-folded protein with an $\alpha$-helical secondary structure. As anticipated, the free peptide in the aqueous solution had a random coil-type spectrum, with a negative peak at $203 \mathrm{~nm} .{ }^{21,31,44,46}$ Adding 1 equivalent of XPC to the C-EoCen solution induced a moderate enhancement (by $26 \%$ ) in the molar ellipticity, which suggested that the peptide underwent a random coil-to-helix transition upon binding to the protein. Doubling the peptide-to-protein ratio $(2: 1)$ was not accompanied by obvious CD changes, in perfect agreement with the ITC-obtained $1: 1$ stoichiometry. The restricted conformational freedom associated with the peptide transition should overcome the dehydration effect (related to an increase in water entropy), and accounted for the overall negative (unfavorable) entropy contribution to the free energy of interaction (Table 2).

In the case of the integral protein (Fig. 5B), the addition of the peptide led to a slightly smaller (by $\sim 10 \%$ ) increase in intensity. In contract, there was negligible change when the peptide was added into the N-EoCen solution (Fig. 5C). So, the interaction of EoCen with the XPC peptide was predominantly mediated by the C-terminal fragment while the $\mathrm{N}$-terminal was not involved in complex formation. These findings indicated that the centrin-peptide interaction resulted in well organized, ordered and stable structures.

Table 2 Thermodynamics parameters of the XPC peptide to EoCen and its fragments in the presence of EDTA

\begin{tabular}{lllll}
\hline Protein & $N$ & $K_{\mathrm{a}}(\mathrm{error})\left(10^{6} \mathrm{M}^{-1}\right)$ & $\Delta H(\mathrm{error})\left(\mathrm{kcal} \mathrm{mol}^{-1}\right)$ & $\begin{array}{l}T \Delta S \\
\left(\mathrm{kcal} \mathrm{mol}^{-1}\right)\end{array}$ \\
\hline EoCen & 0.84 & $1.67(0.35)$ & $-14.98(0.25)$ & -6.61 \\
C-EoCen & 0.97 & $0.46(0.09)$ & $-13.88(0.42)$ & -6.15 \\
N-EoCen & $\mathrm{NB}$ & & -8.37 \\
${ }^{a} \mathrm{NB}=$ no binding measured in the present conditions.
\end{tabular}



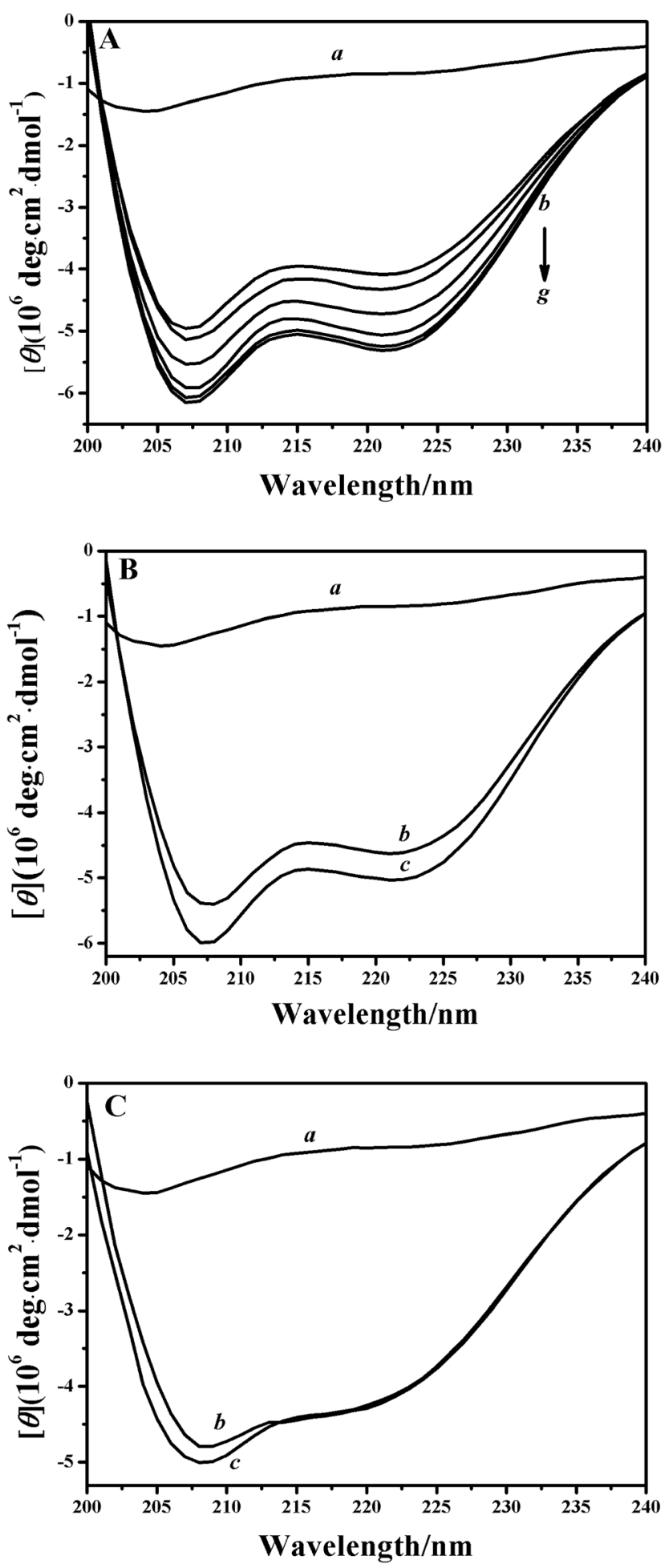

Fig. $5 \mathrm{CD}$ analysis. Far-UV CD spectra of the peptide $(7.84 \mu \mathrm{M})$, integral and fragments of EoCen $(7.84 \mu \mathrm{M})$ in $10 \mathrm{mM}$ Hepes, $100 \mathrm{mM}$ $\mathrm{KCl}(\mathrm{pH} 7.4)$, in the presence of EDTA. The effect of peptide addition to the $\mathrm{C}$-EoCen (the ratio from 0 (b), 0.25 (c), 0.5 (d), 0.75 (e), 1 (f), to 2 (g)) is shown in panel $A$ and that of peptide addition to the EoCen (the ratio of XPC peptide to apoprotein is 0 (b), 1 (c)) is shown in panel B. And no effect of the addition to the $\mathrm{N}$-EoCen (the ratio of $\mathrm{O}(\mathrm{b})$ and 1 (c)) is shown in panel $\mathrm{C}$.
Base on certain physicochemical techniques, we characterized the peptide binding properties of the highly purified EoCen. Exposure of the hydrophobic surface is instrumental for peptide binding. Contrary to CaM, the C-terminal domain of centrin adopted the open conformation even in the absence of $\mathrm{Ca}^{2+}, 49,50$ the N-terminal occupied a closed conformation in the metal-free form and adopted open conformation only in metalsaturated form. Consequently, this may explain the high affinity of C-EoCen to XPC in a metal-free state, while the N-terminal domain is not engaged in peptide recognition. In other words, the binding of EoCen to peptide is $\mathrm{Ca}^{2+}$-independent, although $\mathrm{Ca}^{2+}$ ions could enhance the affinity (unpublished result). This means EoCen can bind to XPC in the absence of $\mathrm{a} \mathrm{Ca}^{2+}$ ion, and trigger the NER system. In the light of the position of the bulk hydrophobic residues that anchor the surface (Fig. 6A), the CaM binding motifs have been grouped into four categories: 1-5-10, 1-8-14, 1-16 and IQ motifs. ${ }^{42,51,52}$ The binding modes of the CaM interaction with its target are described as the canonical wraparound mode, extended mode and dimerization mode. The centrin-binding sequence of XPC is categorized as a 1-4-8 motif (Fig. 6B), a subclass of the 1-14 motif. In addition to the difference in structure between CaM and centrin, the binding sequence of XPC is not long enough to span the two terminals. According to previous work ${ }^{56,57}$ and the data reported in the

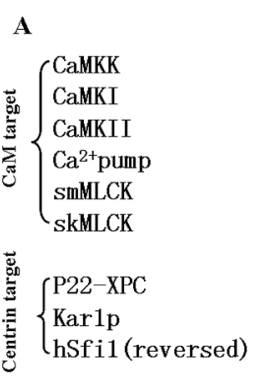

B

\begin{tabular}{|l|r|l|l|l|l|}
\hline HsXPC & 847 & NWKLLAK GLLIRERLKRRYG & 866 \\
\hline MmXPC & 840 & NWKLLVR GLLIRERLKLRYG & 859 \\
\hline DmXPC & 1253 & NWKKLIKGLLIRERLKKKYN & 1272 \\
\hline AtXPC & 762 & RWY WLLS & ILTRERLKNRYA & 781 \\
\hline ScRad4p & 648 & SWNT LLLKLRIRSKLN STYG & 667 \\
\hline
\end{tabular}

Fig. 6 (A) Sequence and motifs of CaM and centrin-binding peptides. Green residues indicate the bulky hydrophobic side chains anchoring the peptide into the hydrophobic pocket of the protein. They define the common patterns for the CaM (top) or centrin (bottom) targets. Blue (basic) and red (acidic) residues are involved in electrostatic interactions with the protein. Note that the sequence of the repeat in hSfi1 (residues 112-131) is reversed. (B) Evolutionary conservation of the amino acid sequences of the putative centrin 2-binding domain among the XPC homologs of human (Hs), mouse (Mm), Drosophila melanogaster (Dm), Arabidopsis thaliana (At), and Saccharomyces cerevisiae (Sc). Identical and similar amino acids with respect to the human sequence are indicated by solid and shaded boxes, respectively. The positions of the three amino acids that are involved in the interaction with centrin 2 are indicated by red boxes. 
A

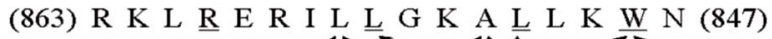

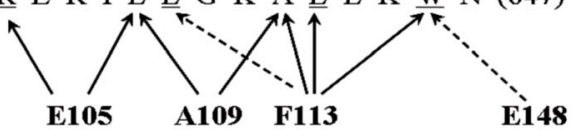

B

C-HsCen2 94 TQKMSEKDTKEEILKAFKLFDDDETGKISFKNLKRVAKEL 133

C-EoCen 90 TEKIKNRDPVEEILKAFKVFDEDNSGKISLRNLKRVAKEL 129

$* * \quad * \quad * * * * * * * * * * * * * * * * * * * * * * * * *$

C-HsCen2 134 GENLTDEELQEMIDEADRDGDGEVSEQEFLRIMKKTSLY 172

C-EoCen 130 GENLSDDELQAMIDEFDKDQDGEISEQEFLNIMKQTSIY 168

$* * * * * * * * * * * * * * * * * * * * * * * * * * * * * * * *$

Fig. 7 The centrin residues involved in target binding. (A) Graph showing the main human centrin 2 residues interacting with the target $\mathrm{XPC}$. The F113 residue is central to the interaction and makes contacts with three conserved residues (underlined) that form the hydrophobic triad of the centrin-target. (B) The amino acid sequence of the Cterminal of human centrin 2 and the $\mathrm{C}$-terminal domain of Euplotes octocarinatus centrin. The two putative EF-hands are highlighted in yellow. Asterisks indicate amino acid identities. Critical amino acids that interact with XPC are shown in red.

work, the binding mode of EoCen to P22-XPC is in the extended mode (Fig. 8).

From fluorescence and UV difference spectra, Trp, which is highly conserved and critical anchoring residue, transfers from the polar water solvent to the apolar hydrophobic surroundings. A complex still could form even in conditions with a high salt concentration. Native PAGE analysis also reflects that the electrostatic component is not a primary force. Two crystal structures of the full-length (2ggm.pdb) ${ }^{53}$ and truncated HsCen2 in complex with an XPC peptide (2obh.pdb) ${ }^{54}$ and NMR structure of C-HsCen2 complexed with XPC peptide (2a4j.pdb) ${ }^{55}$ have been reported. The structure from ref. 53 shows that nine nonpolar residues of HsCen2 (L133, L112, M145, F113, M166, L126, A109, E105 and V129) contact with the triad of the XPC peptide $\left(\mathrm{W}^{1} \mathrm{xxL}^{4} \mathrm{xxxL}^{8}\right)$ and the Trp residue is buried in the hydrophobic pocked created by the C-terminal and two EF-hand motifs (Fig. 7A). Besides, the carboxyl group of E148 established a hydrogen bond with the Trp residue of the XPC peptide. ${ }^{33}$

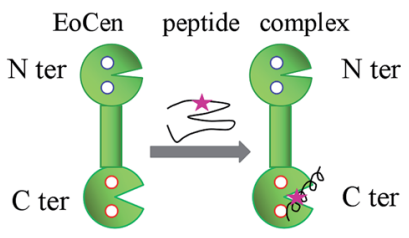

Fig. 8 Binding mode of the XPC peptide to EoCen according to the available data. The $\mathrm{N}$ - and $\mathrm{C}$-terminal domains are shown by two types of circle sectors representing closed and open conformations. The high and low affinity sites for $\mathrm{Ca}^{2+}$ of the EF-hands are shown by red and blue empty circles, respectively. The tryptophan residue in the XPC peptide is shown by the purple star.
Sequence analysis shows that C-EoCen (90T-168Y) shares 71\% sequence identity with C-HsCen2 (94T-172Y), and, what is more, eight non-polar residues that bind to the XPC peptide and E144 residue (corresponding to the $\mathrm{E} 148$ residue in HsCen2) are completely conserved (Fig. 7B), so we surmise that the force between EoCen and the XPC peptide is predominantly a hydrophobic interaction.

The XPC peptide, which is an amphiphilic molecule and exhibits a disordered structure in the absence of the protein, folds into an $\alpha$ helix upon binding to centrin, which induces the structural rearrangement and decrease in flexibility, giving a negative entropic term to the free energy of interaction. The centrin/XPC peptide interaction is driven by a negative enthalpy change. The affinity constants are $1.67 \times 10^{6} \mathrm{M}^{-1}$ and $0.46 \times$ $10^{6} \mathrm{M}^{-1}$ for EoCen and C-EoCen, respectively, by ITC. While the constants are $5.88 \times 10^{6} \mathrm{M}^{-1}$ and $4.55 \times 10^{6} \mathrm{M}^{-1}$ for EoCen and C-EoCen, respectively, by spectrofluorimetric titration. There is little difference in the constants by the two measurement methods. Fluorescence spectroscopy is very sensitive for the tryptophan residue and requires a lower concentration of the sample, whereas ITC needs a higher concentration of the sample and is imprecise during the fitness of data. The reduced affinity by a factor of 4.67 by comparison with HsCen 2 obtained from ITC is the result of at least two main reasons. One is related to the amino acid sequence difference, V108 in EoCen vs. L112 in HsCen2. Holes formed by the Val residue in EoCen differ from those formed by the Leu residue in HsCen2, so the affinity constants vary. In other words, the fitness of interfaces between the key and hole causes the difference in the binding affinity. The side chain of the Val residue lacks one methylene by comparison with the Leu residue. The difference between the Val and Leu residues results in the distinct interface hydrophobicities. The other reason is that the human XPC peptide is not a native target molecule of EoCen. There is only one centrin isoform in Euplotes octocarinatus, so EoCen must participate in the duplication and separation of MTOCs and in the NER process. We offer a glimpse into the centrin function in the cell process with the aid of the human XPC peptide and infer the possibility of NER throughout the lower eukaryotic kingdom, which will motivate us to explore the mechanism further.

\section{Conclusions}

The work represents a structural and thermodynamic characterization of a 22-residue peptide (K842-R863) from human XPC binding to Euplotes octocarinatus centrin using spectroscopic methods, native PAGE, and ITC. Comparisons of the corresponding results for integral protein and isolated fragments show that the residues of the C-terminal are critical for the interaction with the XPC peptide, and the N-terminal domain is structurally independent from the peptide-bound C-terminal domain. Fluorescence and UV difference spectra of the intrinsic Trp manifest the highly conserved Trp residue deeply embed in the hydrophobic cavity formed by the C-terminal domain. CD and ITC suggest that the complex formation results in a more helical content, and a well-organized structure. The new complex was visualized by native PAGE. The 
affinity decreases 4.67 fold in comparison with that of HsCen2. The amino acids that contact with the XPC peptide and the binding mode both resemble those of HsCen2. The only difference lies in V108 in the EoCen substitution for L112 in HsCen2. The distinction of the interface hydrophobicity and the fitness of interfaces between the key and hole causes the difference in the binding affinity.

\section{Acknowledgements}

The work was supported by the National Natural Science Foundation of the PR China (No. 21571117) and the Ph.D. Programs Foundation of the Ministry of Education of China (20131401110011). We thank Scientific Instrument Center of Shanxi University for the help with ITC measurements.

\section{References}

1 J. L. Salisbury, A. Baron, B. Surek and M. Melkonian, J. Cell Biol., 1984, 99, 962-970.

2 B. Huang, A. Mengersen and V. D. Lee, J. Cell Biol., 1988, 107, 133-140.

3 M. A. Sanders and J. L. Salisbury, J. Cell Biol., 1994, 124, 795805.

4 P. Baum, C. Furlong and B. Byers, Proc. Natl. Acad. Sci. U. S. A., 1986, 83, 5512-5516.

5 J. K. Zhu, R. A. Bressan and P. M. Hasegawa, Plant Physiol., 1992, 99, 1734-1735.

6 U. Wolfrum, Biol. Cell., 1992, 76, 373-381.

7 V. D. Lee and B. Huang, Proc. Natl. Acad. Sci. U. S. A., 1993, 90, 373-381.

8 R. Errabolu, M. A. Sanders and J. L. Salisbury, J. Cell Sci., 1994, 107, 9-16.

9 A. Paoletti, M. Moudjou, M. Paintrand, J. L. Salisbury and M. Bornens, J. Cell Sci., 1996, 109, 3089-3102.

10 J. L. Salisbury, K. M. Suino, R. Busby and M. Springett, Curr. Biol., 2002, 12, 1287-1292.

11 S. Middendorp, T. Küntziger, Y. Abraham, S. Holmes, N. Bordes, M. Paintrand, A. Paoletti and M. Bornens, J. Cell Biol., 2000, 148, 405-416.

12 M. Araki, C. Masutani, M. Takemura, A. Uchida, K. Sugasawa, J. Kondoh, Y. Ohkuma and F. Hanaoka, J. Biol. Chem., 2001, 276, 18665-18672.

13 R. Nishi, Y. Okuda, E. Watanabe, T. Mori, S. Iwai, C. Masutani, K. Sugasawa and F. Hanaoka, Mol. Cell. Biol., 2005, 25, 5664-5674.

14 T. Fischer, S. Rodríguez-Navarro, G. Pereira, A. Rácz, E. Schiebel and E. Hurt, Nat. Cell Biol., 2004, 6, 840-848.

15 A. Giessl, A. Pulvermüller, P. Trojan, J. H. Park, H. W. Choe, O. P. Ernst, K. P. Hofmann and U. Wolfrum, J. Biol. Chem., 2004, 279, 51472-51481.

16 K. Gonda, A. Yoshida, K. Oami and M. Takahashi, Biochem. Biophys. Res. Commun., 2004, 323, 891-897.

17 S. L. Jaspersen, T. H. Giddings and M. Winey, J. Cell Biol., 2002, 159, 945-956.

18 J. V. Kilmartin, J. Cell Biol., 2003, 162, 1211-1221.
19 D. Jani, S. Lutz, N. J. Marshall, T. Fischer, A. Köhler, A. M. Ellisdon, E. Hurt and M. Stewart, Mol. Cell, 2009, 33, 727-737.

20 I. Durussel, Y. Blouquit, S. Middendorp, C. T. Craescu and J. A. Cox, FEBS Lett., 2000, 472, 208-212.

21 J. A. Cox, F. Tirone, I. Durussel, C. Firanescu, Y. Blouquit, P. Duchambon and C. T. Craescu, Biochemistry, 2005, 44, 840-850.

22 Y. Q. Zhao, J. Y. Feng, A. H. Liang and B. S. Yang, Chin. Sci. Bull., 2007, 52, 3216-3220.

23 Y. Q. Zhao, J. Y. Feng, Z. J. Wang, A. H. Liang and B. S. Yang, Spectrochim. Acta, Part A, 2008, 70, 884-887.

24 X. J. He, J. Y. Feng, W. Wang, B. F. Chai, B. S. Yang and A. H. Liang, Acta Zool. Sin., 2004, 50, 447-451.

25 C. Weber, V. D. Lee, W. J. Chazin and B. Huang, J. Biol. Chem., 1994, 269, 15795-15802.

26 A. Yang, S. Miron, P. Duchambon, L. Assairi, Y. Blouquit and C. T. Craescu, Biochemistry, 2006, 45, 880-889.

27 M. Tourbez, C. Firanescu, A. Yang, L. Unipan, P. Duchambon, Y. Blouquit and C. T. Craescu, J. Biol. Chem., 2004, 279, 47672-47680.

28 H. Wiech, B. M. Geier, T. Paschke, A. Spang, K. Grein, J. Steinkötter, M. Melkonian and E. Schiebel, J. Biol. Chem., 1996, 271, 22453-22461.

29 L. Duan, Y. Q. Zhao, Z. J. Wang, G. T. Li, A. H. Liang and B. S. Yang, J. Inorg. Biochem., 2008, 102, 268-277.

30 Y. Q. Zhao, L. Song, A. H. Liang and B. S. Yang, J. Photochem. Photobiol., B, 2009, 95, 26-32.

31 A. Popescu, S. Miron, Y. Blouquit, P. Duchambon, P. Christova and C. T. Craescu, J. Biol. Chem., 2003, 278, 40252-40261.

32 H. T. Hu and W. J. Chazin, J. Mol. Biol., 2003, 330, 473-484.

33 J. Martinez-Sanz, F. Kateb, L. Assairi, Y. Blouquit, G. Bodenhausen, D. Abergel, L. Mouawad and C. T. Craescu, J. Mol. Biol., 2010, 395, 191-204.

34 Y. Q. Zhao, X. L. Diao, J. Yan, Y. N. Feng, Z. J. Wang, A. H. Liang and B. S. Yang, J. Lumin., 2012, 132, 924-930.

35 K. Sugasawa, J. M. Ng, C. Masutani, S. Iwai, P. J. van der Spek, A. P. Eker, F. Hanaoka, D. Bootsma and J. H. J. Hoeijmakers, Mol. Cell, 1998, 2, 223-232.

36 L. Liang, S. Flury, V. Kalck, B. Hohn and J. Molinier, Plant Mol. Biol., 2006, 61, 345-356.

37 Y. S. Krasikova, N. I. Rechkunova, E. A. Maltseva, P. E. Pestryakov, I. O. Petruseva, K. Sugasawa, X. J. Chen, J.-H. Min and O. I. Lavrik, J. Biol. Chem., 2013, 288, 1093610947.

38 B. den Dulk, P. van Eijk, M. de Ruijter, J. A. Brandsma and J. Brouwer, DNA Repair, 2008, 7, 858-868.

39 J. H. Park, A. Pulvermüller, P. Scheerer, S. Rausch, A. Gießl, W. Höhne, U. Wolfrum, K. P. Hofmann, O. P. Ernst, H.-W. Choe and N. Krauß, Vision Res., 2006, 46, 4568-4574.

40 B. Wang, S. R. Martin, R. A. Newman, S. L. Hamilton, M. A. Shea, P. M. Bayley and K. M. Beckingham, Protein Sci., 2004, 13, 3285-3297.

41 E. A. Burstein, N. S. Vedenkina and M. N. Ivkova, Photochem. Photobiol., 1973, 18, 263-279. 
42 A. Crivici and M. Ikura, Annu. Rev. Biophys. Biomol. Struct., 1995, 24, 85-116.

43 B. M. Geier, H. Wiech and E. Schiebel, J. Biol. Chem., 1996, 271, 28366-28374.

44 J. Martinez-Sanz, A. Yang, Y. Blouquit, P. Duchambon, L. Assairi and C. T. Craescu, FEBS J., 2006, 273, 4504-4515.

45 H. T. Hu, J. H. Sheehan and W. J. Chazin, J. Biol. Chem., 2004, 279, 50895-50903.

46 J. F. Maune, C. B. Klee and K. Beckingham, J. Biol. Chem., 1992, 267, 5286-5295.

47 Z. J. Wang, Y. Q. Zhao, L. X. Ren, G. T. Li, A. H. Liang and B. S. Yang, J. Photochem. Photobiol., A, 2007, 186, 178-186.

48 W. C. Johnson, Proteins: Struct., Funct., Genet., 1999, 35, 307312.

49 E. Matei, S. Miron, Y. Blouquit, P. Duchambon, I. Durusse, J. A. Cox and C. T. Craescu, Biochemistry, 2003, 42, 14391450 .
50 S. Veeraraghavan, P. A. Fagan, H. T. Hu, V. Lee, J. F. Harper, B. Huang and W. J. Chazin, J. Biol. Chem., 2002, 277, 2856428571.

51 A. R. Rhoads and F. Friedberg, FASEB J., 1997, 11, 331-340.

52 K. L. Yap, J. Kim, K. Truong, M. Sherman, T. Yuan and M. Ikura, J. Struct. Funct. Genomics, 2000, 1, 8-14.

53 J. R. Thompson, Z. C. Cyan, J. L. Salisbury and R. Kumar, J. Biol. Chem., 2006, 281, 18746-18752.

54 J. B. Charbonnier, E. Renaud, S. Miron, M. H. Le Du, Y. Blouquit, P. Duchambon, P. Christova, A. Shosheva, T. Rose, J. F. Angulo and C. T. Craescu, J. Mol. Biol., 2007, 373, 1032-1046.

55 A. Yang, S. Miron, L. Mouawad, P. Duchambon, Y. Blouquit and C. T. Craescu, Biochemistry, 2006, 45, 3653-3663.

56 L. X. Ren, Y. Q. Zhao, J. Y. Feng, X. J. He, A. H. Liang and B. S. Yang, Chin. J. Inorg. Chem., 2006, 22, 87-90.

57 Y. Q. Zhao, J. Y. Feng, A. H. Liang and B. S. Yang, Spectrochim. Acta, Part A, 2009, 71, 1756-1761. 